\title{
Estimation of gait normality index based on point clouds through deep auto-encoder

\author{
Trong-Nguyen Nguyen ${ }^{*}$ (D) and Jean Meunier ${ }^{1}$
}

\begin{abstract}
This paper proposes a method estimating an index that indicates human gait normality based on a sequence of 3D point clouds representing the walking motion of a subject. A cylinder-based histogram is extracted from each cloud to reduce the number of data dimensions as well as highlight gait-related characteristics. A model of deep neural network is finally formed from such histograms of normal gait patterns to provide gait normality indices supporting gait assessment tasks. The ability of our approach is demonstrated using a dataset of 9 different gait types performed by 9 subjects and two other datasets converted from mocap data. The experimental results are also compared with other related methods that process different input data types including silhouette, depth map, and skeleton as well as state-of-the-art deep learning approaches working on point cloud.
\end{abstract}

Keywords: Gait, Normality index, Auto-encoder, Deep network

\section{Introduction}

Gait normality index estimation is one of the most common studied problems to support healthcare systems. Many researchers employed complex marker-based and multi-camera systems to acquire more details for gait analysis. One of their drawbacks is that they require specific devices with high price and/or have high computational cost. Therefore, some recent studies employed a single camera to deal with gait analysis problems. Depending on the used sensors, the input of those methods is either subject's silhouette or depth map. The former information has been used to propose numerous gait signatures such as motion history image (MHI) [9], gait energy image (GEI) [11], and active energy image (AEI) [17]. Each signature is a compression of a sequence of consecutive $2 \mathrm{D}$ silhouettes and is represented as a single grayscale or binary image. They were usually applied for the task of person identification. However, in the case of gait normality index estimation, using only the gait signature is not enough. Nguyen et al. [20] employed MHI to estimate four-dimensional features. They processed each individual silhouette as well as segmented each input sequence of frames into gait cycles where the temporal context

*Correspondence: nguyetn@iro.umontreal.ca

'DIRO, University of Montreal, Pavillon André-Aisenstadt, 2920 chemin de la Tour, Montreal, H3T 1 J4 Canada was embedded in. The gait assessment was performed on each gait cycle using a one-class model that was trained with normal gait patterns, i.e., unsupervised learning. Bauckhage et al. [4] also proposed an approach detecting unusual movement. They put a camera to capture the frontal view of a walking subject. Each silhouette was encoded by a flexible lattice that followed a vector conversion of coordinates corresponding to a set of predefined control points. The temporal characteristic was then integrated into each feature vector by concatenating vectors of consecutive frames. Differently from [20], the gait normality decision was determined based on a binary SVM where both normal and abnormal gait samples appeared in the training set. However, in many applications, using only a sequence of silhouettes as the input would lose important gait information because of the missing depth.

In order to deal with that limitation, depth sensors replaced color cameras in some studies. A popular device is the Kinect, which is provided by Microsoft with a low price and a SDK containing the functionality of perframe 3D human skeleton localization [29, 30]. Such skeletons played the main role in some recent studies of gait-related problems such as pathological gait analysis [8], gait recognition [16], and abnormal gait detection [21]. These approaches, however, still have a drawback since each skeleton is determined based on a depth frame. Concretely, self-occlusions in depth maps might 
lead to unusual skeleton postures, and embedded gait characteristics would thus be deformed.

In this paper, we present an approach dealing with the problem of gait normality estimation. We focus on a setup of cheap equipments to capture the motion from different view points. We employ a time-of-flight (ToF) depth camera together with two mirrors so that the system can work in the manner of a collection of cameras while keeping the cost much lower than multi-camera systems [22]. A subject performs her/his walking gait on a treadmill at the center of the setup. A depth map captured by our setup is presented in Fig. 1. As shown in the figure, there are 3 regions (highlighted with ellipses) corresponding to partial subject's surfaces seen from different view points. A point cloud representing the subject can thus be easily formed as a combination of 3 collections of reprojected points (from 2D to 3D) including (a) the real cloud in the middle and (b) reflections (through mirror planes) of virtual clouds that are behind the two mirrors. An example of such reconstructed 3D point cloud is presented in Fig. 2. More details on this reconstruction method are given in [22]. The input of our method is a sequence of these 3D point clouds that are formed based on consecutive depth frames captured by the depth camera. The output is gait normality indices provided by a model of normal walking postures. To our knowledge, this is the first work that performs gait normality index estimation on a sequence of 3D point clouds representing a walking person.

Our contributions are summarized as follows:

- Proposing a deep auto-encoder that learns common features of gait normality based on histograms of

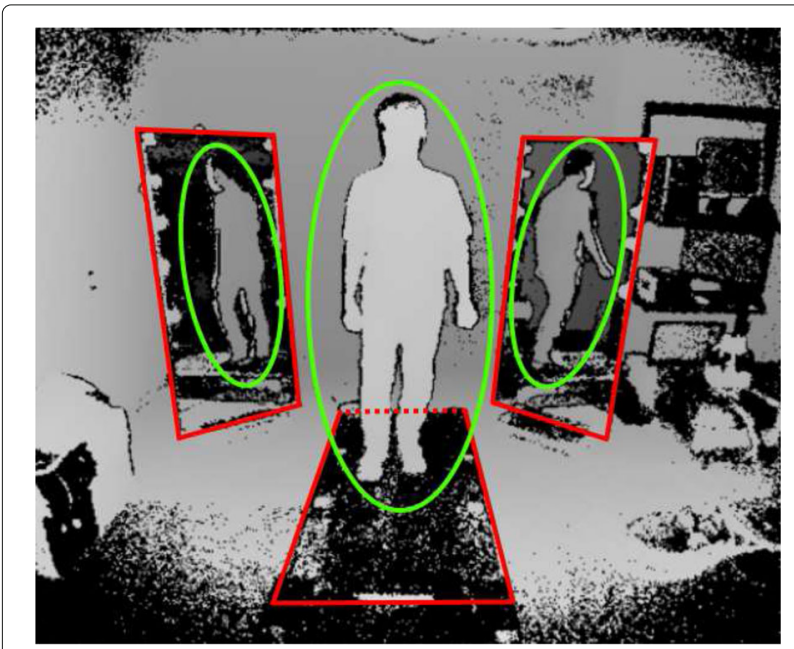

Fig. 1 A depth map captured by our setup that shows 3 devices including two mirrors and a treadmill where each subject performs her/his walking gait. Three collections of subject's pixels are highlighted by ellipses point clouds and a discussion on cloud-oriented deep networks for gait analysis

- Demonstrating the potential of point cloud in gait analysis problems compared to typical input data types such as skeleton, depth map, and silhouette

\section{Proposed method}

Our method consists of three main steps. First, a 2D histogram of each point cloud is formed to normalize the data dimension as well as highlight gait-related characteristics. Then, the second stage generates a model representing postures corresponding to normal walking gait based on a collection of 2D histograms. Finally, this model is used to compute a normality index for gait analysis.

\subsection{Cylindrical histogram estimation}

There are some inconveniences when performing gait assessment on 3D point clouds: (1) the number of points inside each cloud is not normalized, (2) such cloud may contain redundant information that are not useful for gaitrelated tasks, and (3) there may be some noises in each cloud, i.e., points reconstructed from depth values containing noise in the depth map. Therefore, each $3 \mathrm{D}$ point cloud is converted into a $2 \mathrm{D}$ histogram by fitting a cylinder with equal sectors. It is worth noting that this step of normalization also plays an important role when working with neural networks since such models require inputs of fixed dimensions. Its axis coincides with the normal vector of the treadmill surface and goes through the cloud's centroid. Illustrations of the cylinder fitting and histogram formation are shown in Fig. 3.

Let us notice that the coordinate system in that figure is flexible. The only constraint is that the $y$-axis must be normal to the treadmill surface. The coordinate system in Fig. 3 is to show the relation between cylindrical sectors and their mapped elements in the corresponding 2D histogram. Such arrangement of elements inside a histogram is to highlight the balance of human posture embedded in the point cloud. In other words, our cylindrical histogram is considered as a smart projection of a $3 \mathrm{D}$ point cloud onto a frontal (or back) grid. The element values of each histogram are finally scaled to give a grayscale image of 256 levels. This representation is convenient for data range normalization and for storing. An example of grayscale histogram and the corresponding human posture is given in Fig. 4.

\subsection{Model of normal gait postures}

Many recent studies embedded the temporal context into features that were then employed to create a model supporting gait classification. Our model, however, considers only individual postures. The temporal factor can then be integrated by extracting statistical quantities based on a 

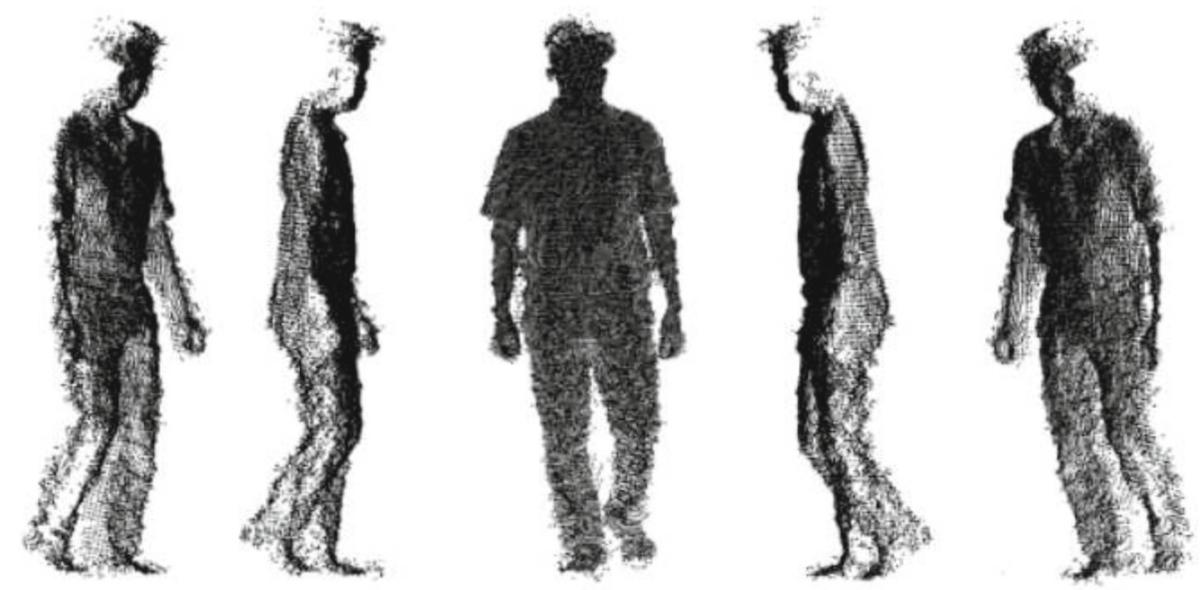

Fig. 2 The point cloud reconstructed from a depth map using the method [22]

sequence of posture assessments. An unsupervised learning is appropriate since we are focusing on estimating gait normality index. A model that is formed from a training set containing both normal and abnormal gaits may have a low generalization. The reason is that patterns of abnormal gaits would significantly affect the classifier because there are too numerous possible types of walking postures with abnormality in practical situations. Therefore, we attempt to create a model describing common characteristics of normal gait postures. A typical way of performing this task is learning a vocabulary of code words extracted from histograms of normal gait. Recently, such approaches have demonstrated good performance on common problems such as content-based image retrieval $[2,42]$ and image classification [3, 40, 41]. Another approach is the use of pretrained deep networks for feature extraction such as [27, 31]. These methods, however, are applied on natural images with an appropriate resolution, in which each code word is formed from an image patch. Therefore, vocabulary learning is not suitable to deal with our histograms of small size $16 \times 16$. Since deep learning has provided very good results in recent studies, we decide to employ such structures that can automatically determine useful features itself and work as a one-class classifier. The deep auto-encoder [26] is thus chosen in our approach to model normal gait postures.

Our model structure is similar to a typical neural network but has some specific constrains. First, the model is a stack of blocks with the same layers inside. The only difference between these blocks is the number of input and output connections. Each block contains a fully connected layer, a nonlinear activation layer, and an optional dropout layer. The dropout layer is considered to reduce the risk of overfitting [32]. We selected 3 popular activation functions including sigmoid, tanh, and leaky ReLU (rectified linear unit) for the middle (or last if no dropout) layer in each block. The original ReLU function is not considered because it may cause the problem of dead neuron [18]

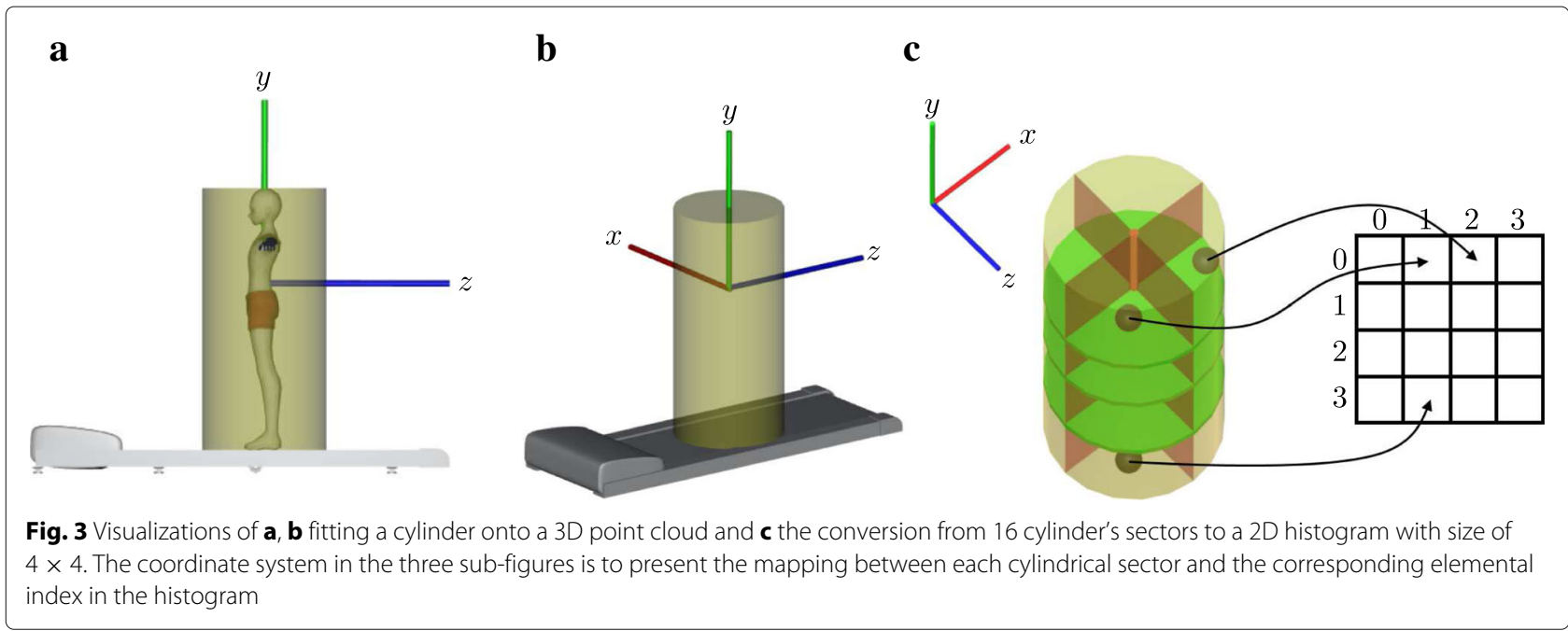




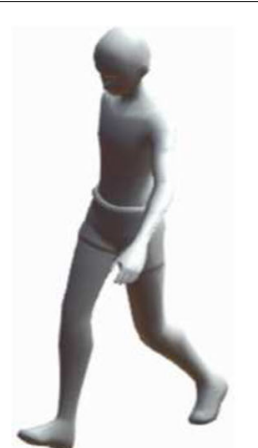

a

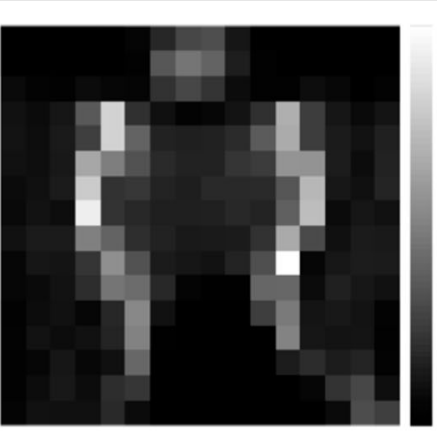

b

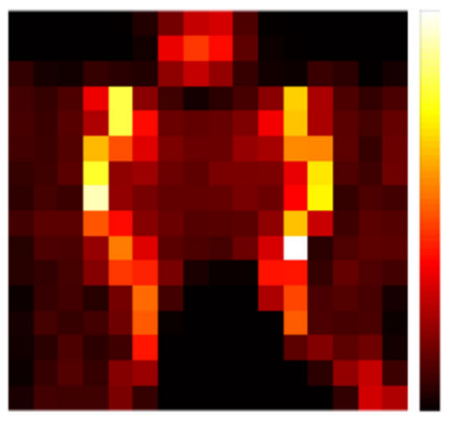

c

Fig. 4 Example of 2D histogram estimated by fitting a cylinder onto a 3D point cloud: a posture, $\mathbf{b}$ grayscale histogram, and $\mathbf{c}$ pseudo-color histogram for better visualization. The size of this histogram is $16 \times 16$

when embedded into a deep fully connected neural network where the learning rate is not small enough.

Let us consider a block $l$ where its fully connected layer is parametrized by weights $W^{(l)}$ and biases $b^{(l)}$, the output of an $i$ th unit given an input $x^{(l)}$ is computed as

$$
\left\{\begin{array}{l}
y_{i}^{(l)}=W_{i}^{(l)} x^{(l)}+b_{i}^{(l)} \\
z_{i}^{(l)}=f\left(y_{i}^{(l)}\right) \\
\hat{z}_{i}^{(l)}=\mathcal{U}_{i}\left(p, N\left(x^{(l)}\right)\right) * z_{i}^{(l)}
\end{array}\right.
$$

where $f$ indicates one of the three mentioned activations, $N\left(x^{(l)}\right)$ is the number of units connected from the previous block, and $\mathcal{U}(p, n)$ is a function that produces $n$ binary values where $p$ is the probability of zero ones. The block output $\hat{z}^{(l)}$ is the input of the next block, i.e., $x^{(l+1)} \leftarrow \hat{z}^{(l)}$.

The second constrain is that when the data is propagated from one block to the next, the number of dimensions is reduced by half. This property is reasonable since auto-encoders are to compress and highlight useful features inside the input. These two constrains are illustrated in Fig. 5. Since we consider one of three activation functions including sigmoid, tanh, and leaky ReLU, there are thus 6 different structures that can be employed for constructing our model. Notice that in the partial network of decoder, the number of units in a next block is doubled but the order of layers inside each block is the same. The auto-encoder structure in our work is symmetric, i.e., we stack $k-1$ blocks with increasing data dimension after using $k$ blocks to encode an input histogram. We use the term block-level depth (or simply depth) to indicate such value of $k$, a model of depth $k$ will thus have $2 k-1$ hidden blocks. The input of our network is a vector of 256 elements that is vectorized from each $16 \times 16$ histogram. The loss function used in our work is the mean squared error
(MSE) combined with a L2-regularization to prevent the model from overfitting:

$$
\mathcal{L}(\mathcal{H}, \hat{\mathcal{H}})=\frac{1}{n} \sum_{i=1}^{n} \frac{\left\|\mathcal{H}_{i}-\hat{\mathcal{H}}_{i}\right\|_{2}^{2}}{256}+\lambda \sum_{l}\left\|W^{(l)}\right\|_{2}^{2}
$$

where $\mathcal{H}$ and $\hat{\mathcal{H}}$ respectively denote a batch of $n$ input vectorized histograms of 256 elements and their reconstruction, $W^{(l)}$ indicates weights of the fully connected layer in block $l$, and $\lambda$ is the regularization rate that controls the effect of $W$ s on the total loss $\mathcal{L}$.

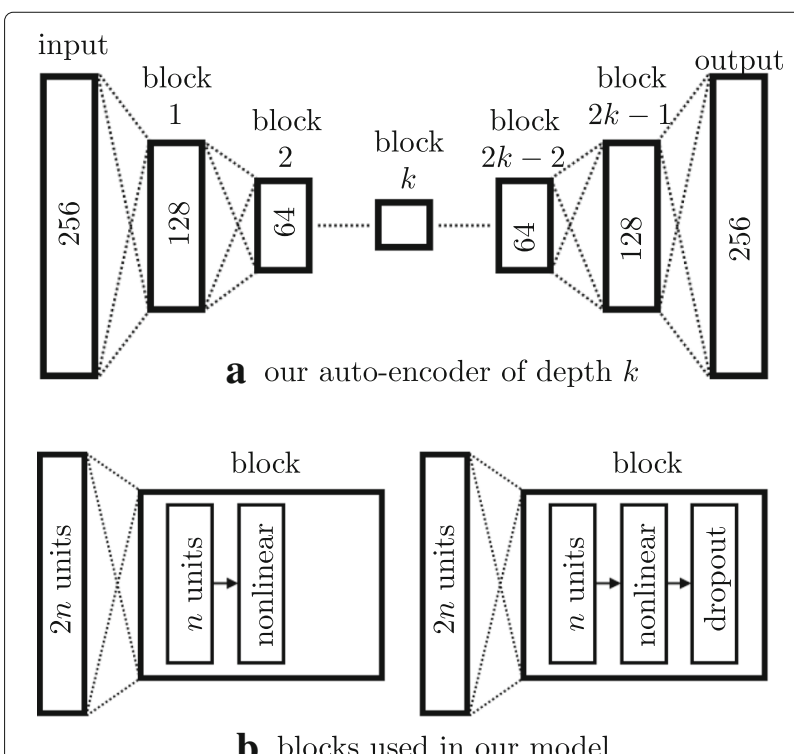

Fig. 5 Structure of our auto-encoder that models characteristics of normal gait postures: $\mathbf{a}$ an example of model of block-level depth $k$ with the number of units indicated inside each block, and $\mathbf{b}$ two possible block structures used in our auto-encoder 


\subsection{Normality index}

Since the input and output of our auto-encoder are the same in the training stage, we expect that the model can learn common characteristics embedded in normal walking gait. We also expect that the loss of information in case of abnormal posture inputs will be significantly higher compared with normal gaits. The normality index is computed for each individual posture as the MSE loss between the input and output vectors of the same size, i.e.,

$$
\mathcal{I}(h)=\frac{1}{256}\|h-\mathcal{M}(h)\|_{2}^{2}
$$

where $h$ is an input vectorized cylindrical histogram and $\mathcal{M}$ denotes the model estimating a reconstruction from $h$. The gait assessment can be performed with or without considering the temporal factor depending on specific problems. Recent studies working on time series data (e.g., action recognition or video retrieval) embedded this factor into their processing in various fashions such as by considering the variance among successive key frames [38], concatenating consecutive frames [35], or using specific neural network layers [36]. In our work, we directly measure a normality index given a sequence of $n$ cylindrical histograms by simply averaging their frame-level indices

$$
\mathcal{I}\left(h_{1 . . n}\right)=\frac{1}{n} \sum_{i=1}^{n} \mathcal{I}\left(h_{i}\right)
$$

This measure is appropriate for the task of gait normality index estimation because of the following reason. A sequence of walking postures can be considered as a hierarchy: it is a collection of walking cycles and each cycle is a group of poses. Unlike related tasks such as action classification or behavior understanding, walking movement tends to be periodic. Given an input sequence that is long enough to cover a number of gait cycles, the average of frame-level normality indices is expected to implicitly indicate the overall measure through the gait cycles.

The details of our model parameters and the ability of measuring gait normality index for distinguishing normal and abnormal walking gaits are shown in the next section.

\section{Experiments}

\subsection{Dataset}

Our approach was experimented on a dataset that includes normal walking gaits and 8 simulated abnormal gaits [19]. The abnormal gaits were created by embedding asymmetry into walking postures. Concretely, this task was performed by one of the following actions: (a) padding a sole with 3 possible heights $(5 / 10 / 15 \mathrm{~cm})$ under the left or right foot or (b) attaching a 4-kg weight to the left or right ankle. There are thus 8 possible walking gaits with anomaly. The normal and abnormal gaits were performed by 9 volunteers using a Kinect 2 . Each gait was represented by a sequence of 1200 consecutive point clouds. They were formed by applying the method proposed in [22] at a frame rate of $13 \mathrm{fps}$. The speed of the treadmill was set at $1.28 \mathrm{kph}$. Beside $3 \mathrm{D}$ point clouds, our data acquisition also captured corresponding skeletons and silhouettes using existing functionalities in the Kinect SDK. These two data types were employed for a comparison between our method and two other related studies. In summary, the dataset contains 1200 point clouds, 1200 silhouettes, and 1200 skeletons for each gait type of a subject. Our experimental procedures involving human subjects were approved by the Institutional Review Board (IRB). The experiments focus on assessing the efficiency of the proposed models and demonstrating the potential of point cloud in gait normality index estimation compared with typical inputs such as skeleton, silhouette, and depth map.

The dataset was split into two sets according to the suggestion in [19]. The first one including gaits of 5 subjects was used in the training stage. The gaits of the 4 remaining subjects were tested to evaluate the ability of our trained models. The same split was also used in our experiments on related works in order to provide a comparison. Beside that data separation, the leave-one-out cross validation (on subject) was also considered to evaluate our method in a more general fashion.

\subsection{Auto-encoder hyperparameters}

This section presents our selection for typical hyperparameters and the strategy for finding a reasonable value for the block-level depth $k$ of our auto-encoder.

\subsubsection{Typical hyperparameters}

First, we consider the algorithm that performs the weight update after each iteration. We employed the RMSProp [33] since the learning rate is adaptively changed instead of being a constant value. An initial learning rate of 0.0001 was thus reasonable. The momentum that controls convergence speed was set to 0.9 according to the suggestion in [33].

Such selection of learning rate leads to the choice of the constant that affects the negative slope of the element-wise nonlinear activation leaky ReLU, i.e., $\alpha$ in the equation $f(x)=1(x<0)(\alpha x)+1(x \geq 0)(x)$. This parameter was set to 0.1 in our model because a too small value (such as 0.01) still sometimes causes the problem of dead neuron.

Another layer that also requires a predefined parameter is dropout. In our model, the probability of forcing input elements to zero was set to 0.3. Using a larger value may cause difficulties for the model in attempting to recover meaningful information during iterations in the training stage. 
The $\lambda$ coefficient controlling the L2-regularization was set to 0.25 after evaluating some randomized generating values. For the training process, we used a batch size of 512 and 800 epochs for each possible network without dropout layer. The number of epochs used for training the models with dropout was higher (1600 in our work) as suggested in [32]. The model weights were initialized according to the method proposed by [10]. Many traditional auto-encoders initialized their weights based on greedy layer-wise pre-training $[7,13]$. Our model, however, is considered as a typical deep neural network where the input is a hand-crafting feature, our selection of weight initialization is thus reasonable. The collection of such hyperparameters is summarized in Table 1.

\subsubsection{Depth determination}

An important factor that is not considered in the previous section is the block-level depth of network [i.e., $k$ in Fig. 5a]. This is the last parameter which needs to be determined in order to form a specific network structure. We selected an appropriate value using a cross-validation strategy applied on the training data consisting of gaits of 5 subjects.

Concretely, the cross-validation was performed with 5 folds, in which each one corresponds to the gaits of a subject. For each value $k$, we tested 6 networks [3 nonlinear activations with/without dropout layer]. Since an auto-encoder is considered as a lossy compression, it is obvious that increasing the number of blocks will increase the loss, i.e., the distance between an input and its reconstructed image. Therefore, we need a more meaningful criterion for depth selection instead of simply performing a loss comparison. Let us recall that our auto-encoder would be trained with the goal of modeling normal walking gait, and the ability of providing gait indices that can well distinguish normal and abnormal gaits is thus suitable for assessing the optimal value of $k$. For a problem of binary decision, the area under curve (AUC) of a receiver operating characteristic (ROC) curve is an appropriate measurement and was used here.

The stage of our fivefold cross-validation was performed as follows. Given a block-level depth value $k_{0}$, we constructed 6 networks with $2 k_{0}-1$ hidden blocks. Each network would provide 5 applicable models since the training data was separated into 5 folds. Each model was trained with the normal gaits of 4 folds (4800 histograms) to get a collection of 10800 MSE loss values when evaluating both normal and abnormal gaits (1200 and 9600 frames, respectively) of the remaining fold. A visualization of this separation is shown in Fig. 6. An AUC was finally estimated from such sequence of losses to represent the model's ability. Therefore, each of the 6 networks provided 5 AUCs in the stage of cross-validation given a specific depth. The mean AUC was calculated to represent the strength of each network for different depths in Fig. 7. Notice that we did not consider the choice of block structure, the cross-validation is just to find a reasonable depth for our auto-encoders.

According to Fig. 7, assigning 4 as the network blocklevel depth is a good choice since it provided the highest mean AUC and a relatively small standard deviation (that can be considered as a stability criterion). Our final network was thus trained with 7 hidden blocks (i.e., depth of 4) with hyperparameters in Table 1 using all normal gaits in the training data. The overall architecture of our model can be represented as a sequence of blocks F128ADF64AD-F32AD-F16AD-F32AD-F64AD-F128AD-F256, in which FxAD indicates a block where $\mathrm{F}$ is a fully connected layer that outputs $x$ units, $\mathrm{A}$ is a nonlinear activation (sigmoid, tanh, or leaky ReLU), and D is a dropout layer. When performing experiments on the models of non-dropout blocks, we simply set the dropout probability to 0 .

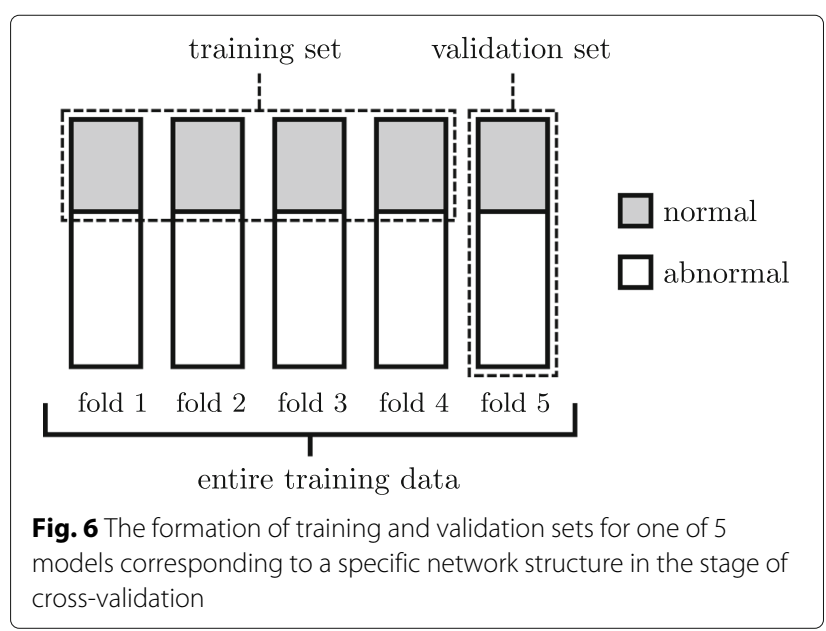

Table 1 Empirically selected hyperparameters in our auto-encoders

\begin{tabular}{ll}
$\begin{array}{l}\text { Table } 1 \text { Empirically selected hyperparameters in our } \\
\text { auto-encoders }\end{array}$ \\
\hline Training algorithm & RMSProp \\
Loss function & MSE \\
Initial learning rate & 0.0001 \\
$\lambda$ (L2-regularization) & 0.25 \\
Momentum & 0.9 \\
Batch size & 512 \\
$\alpha$ (leaky ReLU) & 0.1 \\
Number of epochs (without dropout) & 800 \\
Number of epochs (with dropout) & 1600 \\
Dropout probability & 0.3 \\
Weight initialization & Xavier [10]
\end{tabular}




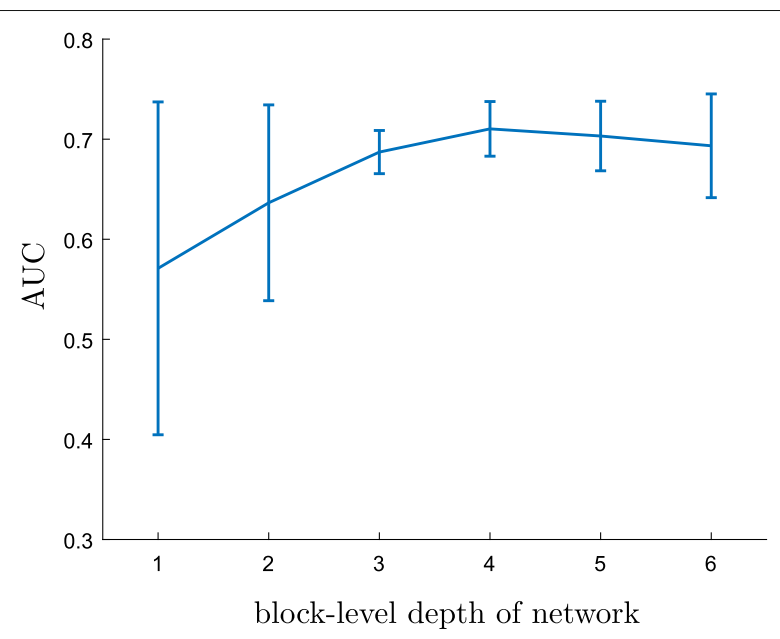

Fig. 7 AUCs estimated in our cross-validation stage with different choices of network depth

There were 6 possible auto-encoders corresponding to 6 block structures. They were employed independently in our evaluations. Our networks were implemented in Python with the use of TensorFlow [1].

\subsection{Reimplementation of related methods}

In order to provide a comparison with other related works that employed different input data types, we also performed experiments on skeletons and silhouettes using the methods proposed in [21] and [5], respectively. The recent study [23] was also considered since it represents features of interest as an intermediate between 2D (silhouette) and 3D (depth map) information. Let us describe briefly these three approaches. The researchers in [21] directly employed the position of lower limb joints in skeletons provided by a Kinect to extract feature vectors representing the subject's walking postures. A sequence of such vectors was then converted into a sequence of codewords using a clustering technique in order to simplify the feature space. The sequence was segmented into gait cycles by considering the change of distance between two feet. This step is necessary since the researchers focused on building a model of normal walking gait cycles using a specific Hidden Markov Model (HMM) structure. The gait normality index was finally estimated for each input cycle as the log-likelihood provided by the trained HMM. Similarly to [21], the authors in [5] also performed the feature extraction on each silhouette using a lattice and embedded the temporal factor by concatenating vectors estimated from a number of consecutive frames. A difference of this method from [21] and ours is that the researchers employed a supervised learning (binary support vector machine (SVM)) with two-class training dataset to distinguish normal and abnormal walking gaits. The method [23] estimated a gait-related score as a weighted sum of two scores corresponding to $2 \mathrm{D}$ and 3D information. Concretely, the researchers measured a LoPS (level of posture symmetry) score using a crosscorrelation technique to describe the symmetry of $2 \mathrm{D}$ subject's silhouette, and simultaneously employed a HMM to compute a PoI (point of interest) score according to key points determined from the corresponding depth map. A combination of those two scores provided good results in distinguishing between normal and abnormal walking gaits. In our experiments, we reimplemented a HMM of normal walking gait cycle for the study [21], a binary SVM for [5], and a combination model of HMM and crosscorrelation for [23]. We also slightly modified the SVM to create a one-class SVM where the training stage only dealt with samples of normal gaits. These models and ours were trained and evaluated on the same dataset split but with different input types, i.e., point cloud, skeleton, and silhouette. Notice that depth maps for experimenting the study [23] were formed based on a projection of 3D point clouds according to the calibration information.

\subsection{Evaluation metric}

The ability of each proposed network was measured according to an equal error rate (EER) estimated based on the collection of MSE loss values. Since some related works attempted to embed the temporal context into their measurement, we also consider it by computing a simple average EER over a short segment (length of 120 in our experiments) of histograms as well as over the entire sequence (i.e., length of 1200) corresponding to each walking gait. Since we did not focus on selecting the best block structure in this work, the average loss of the 6 networks (with $k=4$ ) was also computed. We also need to consider the measure for comparison since the three related works employed different quantities: the AUC for [21], the classification accuracy for [5], and the EER for [23]. We selected the EER estimated from the ROC curve to represent the evaluation result of all models because this measure is related to both AUC and classification accuracy.

\section{Results}

The experimental results on the suggested data split (5 training subjects and 4 test subjects) and the leave-onesubject-out cross-validation are respectively presented in Tables 2 and 3. The last seven models are proposed in our work, in which the term multi-network indicates the assessment of gait normality indices estimated as the average of the losses resulting from the 6 other models. Notice that the notation segment has different meanings: a subsequence of 120 histograms in our approach, a gait cycle that was automatically determined in [21], a per-frame feature that embedded the temporal context of $\Delta=20$ 
Table 2 Classification errors ( $\approx$ EERs) resulting from experiments on our auto-encoders and related studies with different data types

\begin{tabular}{|c|c|c|c|c|c|}
\hline \multirow{2}{*}{ Model } & \multirow{2}{*}{ Training data } & \multirow{2}{*}{ Data type } & \multicolumn{3}{|c|}{ Classification error (4 test subjects) ${ }^{\dagger}$} \\
\hline & & & Per-frame & Segment & Entire seq. \\
\hline HMM [21] & Normal only & Skeleton & - & 0.335 & 0.250 \\
\hline One-class SVM [5] & Normal only & Silhouette & 0.399 & 0.227 & 0.139 \\
\hline Binary SVM [5] & Normal + abnormal & Silhouette & 0.104 & 0.157 & 0.139 \\
\hline HMM [23] & Normal only & Depth map & - & 0.396 & 0.281 \\
\hline Cross-correlation [23] & Normal only & Silhouette & - & 0.381 & 0.250 \\
\hline HMM + cross-correlation [23] & Normal only & Silhouette + depth map & - & 0.377 & 0.218 \\
\hline (Our) Sigmoid & Normal only & Point cloud & 0.332 & 0.264 & 0.250 \\
\hline (Our) Sigmoid + dropout & Normal only & Point cloud & 0.328 & 0.261 & 0.250 \\
\hline (Our) Tanh & Normal only & Point cloud & 0.298 & 0.158 & 0.111 \\
\hline (Our) Tanh + dropout & Normal only & Point cloud & 0.289 & 0.136 & 0.111 \\
\hline (Our) Leaky ReLU & Normal only & Point cloud & 0.326 & 0.125 & 0.028 \\
\hline (Our) Leaky ReLU + dropout & Normal only & Point cloud & 0.296 & 0.103 & 0.028 \\
\hline (Our) Multi-network & Normal only & Point cloud & 0.288 & 0.125 & 0.083 \\
\hline
\end{tabular}

${ }^{\dagger}$ Our system was originally implemented in Mathematica [37]. The models without dropout provided better results compared with the ones performed by TensorFlow [1] in this table. This may be because of the underlying algorithm implementation

The italic values indicate the best results in different evaluations

recent frames in [5], and $\Delta=9$ recent frames in [23]. These values were suggested by the authors in their original works. The term entire sequence indicates EERs calculated based on the average loss over 1200 histograms in our method, lowest mean of log-likelihoods estimated on 3 consecutive walking cycles of a sequence in [21], alarm triggers in [5], and the average score in [23].

According to Tables 2 and 3, employing the temporal factor improved the accuracy in estimating the gait normality index compared with per-frame (i.e., without considering recent frames) estimation except for the binary SVM which is a supervised learning. Therefore, we should focus only on the assessment performed on segment and entire sequence. The classification errors almost always significantly decreased when the gait normality index was estimated over the input sequence instead of short segments. Let us notice that our method measures the index of a sequence as a simple average of per-frame losses while the studies [5] and [21] used nonlinear computations, i.e., decisions respectively based on triggers and minimum 3 -cycles means of log-likelihoods. In other words, those two methods assume that segment-based estimation possibly contains noises (or outliers), a post-processing is thus required to provide a decision. Our method directly

Table 3 Average classification errors ( $\approx$ EERs) resulting from our leave-one-subject-out cross validation

\begin{tabular}{|c|c|c|c|c|c|}
\hline \multirow{2}{*}{ Model } & \multirow{2}{*}{ Training data } & \multirow{2}{*}{ Data type } & \multicolumn{3}{|c|}{ Classification error (leave-one-out) } \\
\hline & & & Per-frame & Segment & Entire seq. \\
\hline $\mathrm{HMM}[21]$ & Normal only & Skeleton & - & 0.396 & 0.198 \\
\hline One-class SVM [5] & Normal only & Silhouette & 0.418 & 0.274 & 0.136 \\
\hline Binary SVM [5] & Normal + abnormal & Silhouette & 0.110 & 0.152 & 0.111 \\
\hline HMM [23] & Normal only & Depth map & - & 0.473 & 0.431 \\
\hline Cross-correlation [23] & Normal only & Silhouette & - & 0.321 & 0.097 \\
\hline HMM + cross-correlation [23] & Normal only & Silhouette + depth map & - & 0.319 & 0.083 \\
\hline (Our) Sigmoid & Normal only & Point cloud & 0.362 & 0.240 & 0.160 \\
\hline (Our) Sigmoid + dropout & Normal only & Point cloud & 0.363 & 0.241 & 0.148 \\
\hline (Our) Tanh & Normal only & Point cloud & 0.298 & 0.144 & 0.049 \\
\hline (Our) Tanh + dropout & Normal only & Point cloud & 0.301 & 0.168 & 0.074 \\
\hline (Our) Leaky ReLU & Normal only & Point cloud & 0.297 & 0.173 & 0.099 \\
\hline (Our) Leaky ReLU + dropout & Normal only & Point cloud & 0.311 & 0.185 & 0.123 \\
\hline (Our) Multi-network & Normal only & Point cloud & 0.303 & 0.178 & 0.086 \\
\hline
\end{tabular}

The italic values indicate the best results in different evaluations 
calculates the index considering every measured loss. There were also several noticeable factors related to the approach [23]. First, the combination of silhouette and depth map in [23] has a lack of generalization compared with our method. Since our dataset (with 8 abnormal gaits) is an extended version of the one in [23] (without gaits with a 4-kg weight attached to the left or right ankle), Table 2 shows that the system [23] encountered difficulty in distinguishing those two additional abnormal gaits from normal ones. Another possible factor affecting the accuracy of method [23] is the size of training set (5 subjects in our experiments vs. 6 subjects in the original paper [23]). This was clearly demonstrated in Table 3, in which the method [23] provided good results when there were 8 training subjects in each fold. It also showed that the generalization ability of our deep neural network is better compared with the combination of HMM and cross-correlation given a small training set.

In order to demonstrate the effect of the length of input walking postures, i.e. , $n$ in Eq. (4), we provide the assessment on various values of the temporal factor in Fig. 8 . These assessment results of default split and leave-oneout cross-validation schemes were respectively obtained from the models with leaky ReLU and tanh activations that provided best results in Tables 2 and 3. Figure 8 shows that the gait normality index estimation tent to be improved with the increasing number of successive postures. Therefore, estimating gait index on a preassigned sufficiently large number of frames is an appropriate choice besides the typical consideration of walking gait cycle.

\section{Comparison with deep learning models}

With the fast development of deep learning, some networks have been proposed to deal with $3 \mathrm{D}$ point cloud

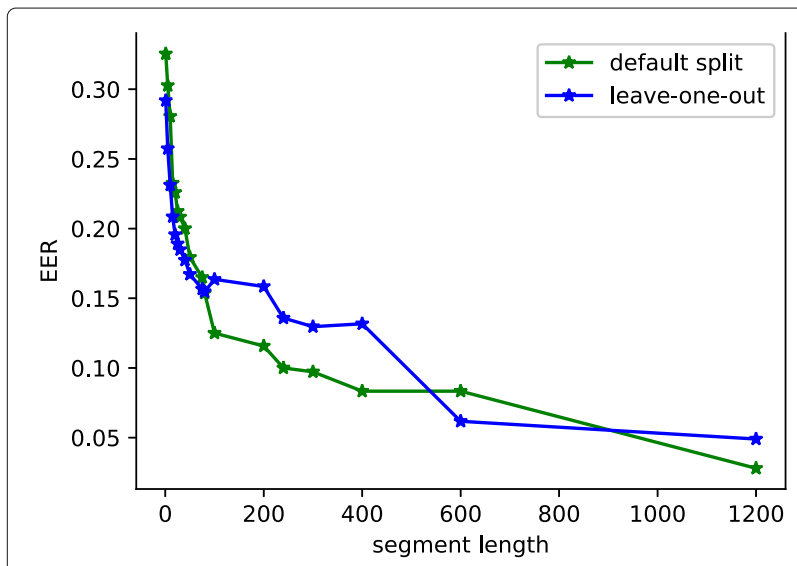

Fig. 8 EERs obtained when the gait normality index was estimated on different lengths of posture sequence for popular objectives such as classification, reconstruction, and segmentation. We adaptively modified ${ }^{1}$ three recent models including FoldingNet [39], PointNet [24], and RSNet [14] to obtain auto-encoder structures supporting the task of gait normality index estimation in the same fashion as ours. The former network is an autoencoder, while the two others are segmentation networks. Details of the reimplementation and experimentation are as follows.

First, each model requires its inputs having the same shape, i.e., a fixed number of points. Therefore, we employed random sampling [34] to downsample the number of points in each input cloud to 2048 for FoldingNet and PointNet and 4096 for RSNet. Second, we adapted the last layer and the objective function of PointNet and RSNet to obtain new architectures of point cloud reconstruction. Concretely, the number of channels in their last layer (corresponding to the number of segmentation categories) was replaced by the number of input channels (i.e., 3 for the coordinates). The softmax loss was changed into MSE loss to force the models learning a way of reconstructing point position instead of performing point classification. The FoldingNet originally uses Chamfer distance for the reconstruction since its input and output clouds have different sizes; we thus did not perform any modification on this model structure. The loss of these models were used to indicate the gait normality index. In order to provide a comparison on processing time, we converted the framework of FoldingNet from Caffe [15] to TensorFlow [1].

Similarly to previous experiments, we evaluated the three networks using two schemes: the suggested data split and the leave-one-subject-out cross-validation. These models were respectively trained for 24000 and 9600 iterations with batch size of 1 for the two schemes. Notice that these numbers of iterations are just to evaluate the potential of models instead of guaranteeing a convergence. We also retrained our best networks (according to Tables 2 and 3) in the same fashion for comparison. Since there was no classification model in this evaluation, we used AUC as the performance measure. The AUCs estimated on the gait indices outputted from all networks are shown in Fig. 9. Notice that we consider only per-frame index.

The experimental results show that our method and FoldingNet have a similar potential for estimating gait normality index. There are some possible reasons for the efficiency of FoldingNet. First, it considers local property of each point via the $k$-NN point-graph and local covariance of its neighborhood. This consideration would thus lead to a good feature extraction/description as typical convolutional neural networks. Second, the reconstructed cloud contains just a small number of outlier points since it is warped from a 2D point grid. Therefore, the use of 


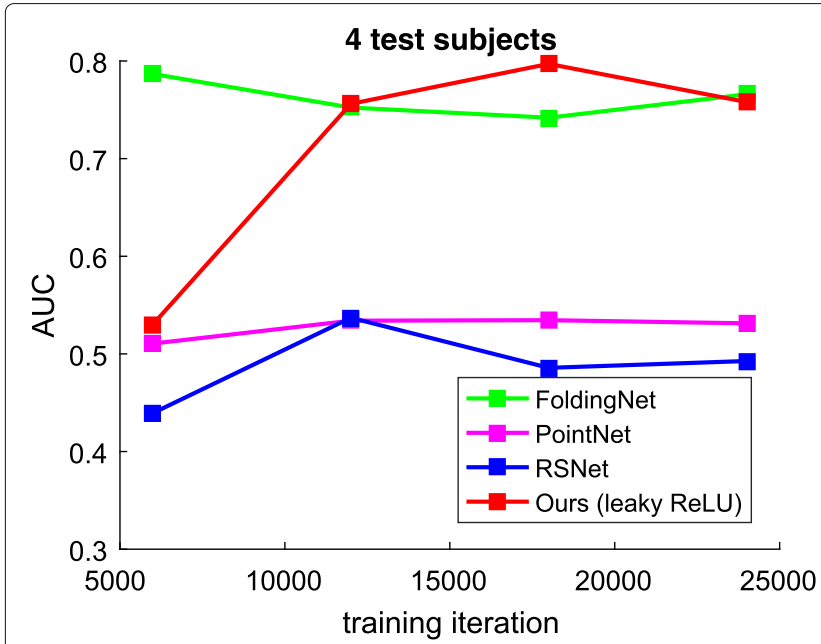

Fig. 9 AUCs estimated from our evaluation on deep learning models

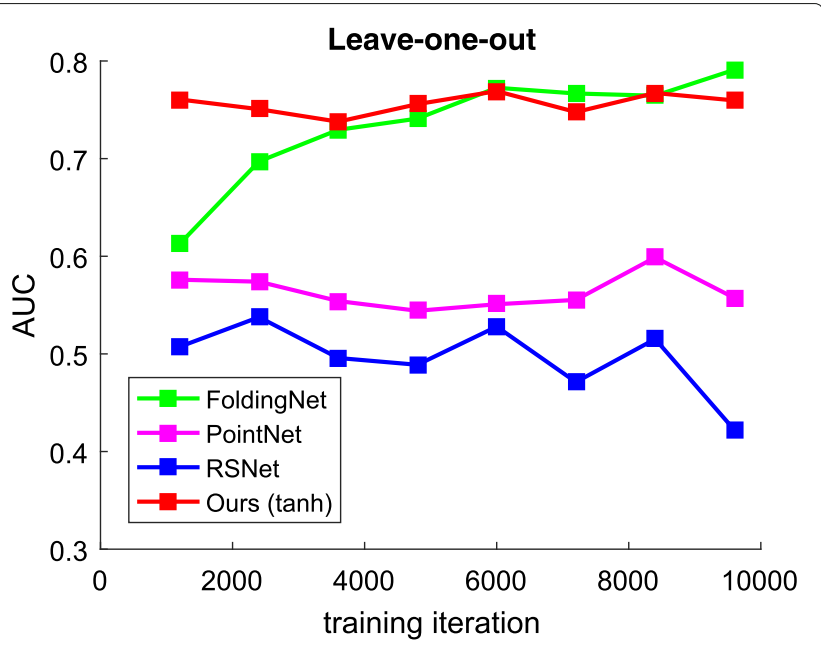

Chamfer distance in gait index calculation is not significantly affected by noise in the input cloud. Recall that there was no enhancement step performed on clouds in our experiments. On the contrary, PointNet and RSNet were directly designed for predicting point's label instead of explicitly emphasizing informative hidden attributes to support the cloud reconstruction. Besides, the point neighborhood is determined using a small network in PointNet and a pooling layer in RSNet while FoldingNet directly considers the distance-based point graph. We believe that this is a reason for the large efficiency gap between FoldingNet and the two others in the task of cloud reconstruction.

A summary of single-cloud processing time corresponding to basic steps in our experiments is given in Table 4 . The evaluation was performed on a single GTX 1080 using Torch 0.4.1 (for RSNet) and TensorFlow 1.10.1 (for the others) with Python 3.5. It is obvious that FoldingNet takes very long times in both training and inference stages compared with our models. This is because we represent each input cloud by a $16 \times 16$ matrix and this size does not increase during propagation in the network. On the contrary, FoldingNet operates on cloud coordinates together with the distance-based graph, performs multiple concatenations, and uses the costly Chamfer distance as the loss function. It should also be noticed that
RSNet may be slightly slower when using TensorFlow since the study [28] reported that Torch is faster than TensorFlow.

\section{Experiments on additional datasets}

In addition to the dataset used for experiments in previous sections, we also performed some testing on two smaller datasets formed from mocap data. In detail, some mocap walking sequences including normal and looking-likeabnormal gaits (unbalance, hobble, skipping, swaggering) were sampled from the $\mathrm{CMU}^{2}$ and $\mathrm{SFU}^{3}$ databases. These mocap data were converted to point clouds by fitting a 3D model (created with MakeHuman ${ }^{4}$ ) and using the set of $3 \mathrm{D}$ vertices as the point clouds. A summary of the two additional datasets used in this experiment is given in Table 5.

In order to provide a comparison, we also reimplemented two recent studies $[6,25]$ that perform gait analysis on human movement. The method [25] decomposes gait input signals into an ensemble of intrinsic mode functions to extract gait frequency properties and then analyzes their association and inherent relations. The study [6] also considers periodical factors, but the gait features were manually estimated from 3D skeletons including average step length, mean gait cycle duration, and leg swing similarity. Both methods focus on efficient gait

Table 4 Average processing time of basic operations in experimented models

\begin{tabular}{lllll}
\hline Model & Framework & Preprocessing (using C++) & Forward and backward (in training stage) & Forward (in inference stage) \\
\hline FoldingNet [39] & TensorFlow & $0.262(\mathrm{~ms})$ & $1.639(\mathrm{~s})$ & $0.446(\mathrm{~s})$ \\
PointNet [24] & TensorFlow & $0.262(\mathrm{~ms})$ & $1.308(\mathrm{~s})$ & $0.102(\mathrm{~s})$ \\
RSNet [14] & Torch & $0.311(\mathrm{~ms})$ & $0.202(\mathrm{~s})$ & $0.058(\mathrm{~s})$ \\
Our 6 models & TensorFlow & $1.126(\mathrm{~ms})$ & $0.014(\mathrm{~s})$ & $0.002(\mathrm{~s})$
\end{tabular}

The preprocessing indicates the cylindrical histogram formation in our method and the cloud downsampling in the others. The time is reported in seconds and milliseconds The italic values correspond to fastest running speeds in execution stages 
Table 5 Number of frames and walking sequences in additional datasets

\begin{tabular}{llcl}
\hline \multirow{2}{*}{ Dataset } & \multirow{2}{*}{ Training set (only normal gait) } & \multicolumn{2}{c}{ Test set } \\
\cline { 3 - 4 } & & Normal & Abnormal \\
\hline CMU & $540(5)$ & $769(8)$ & $2224(7)$ \\
SFU & $1082(5)$ & $1295(6)$ & $3086(13)$ \\
\hline
\end{tabular}

Each pair of values $u(v)$ indicates a collection of $v$ sequences containing a total of $u$ frames

characteristics and employ simple learning algorithms for the assessment.

The experimental results (EER) are presented in Table 6. It shows that our gait normality index was improved over a walking sequence instead of on each frame. Notice that these two datasets were selectively collected from mocap databases focusing on action recognition. Table 6 also shows that the cylindrical histogram can be appropriate for describing various gaits.

\section{Discussion}

First, let us explore in more detail the classification errors provided by the proposed auto-encoders. When embedding the temporal context into the estimation of gait normality index, the model which employed the leaky ReLU activation together with dropout layers provided the best results according to Table 2 . In the leave-oneout cross-validation stage, replacing such combination by $\tanh$ activation gave the lowest classification errors. Therefore, more experiments as well as an extension of the dataset are needed to confirm the best block structure. However, the two tables show that using the tanh and/or leaky ReLU is preferred to sigmoid activation. In addition, the average of indices resulting from the 6 auto-encoders corresponding to 6 block structures (last row of Tables 2 and 3) demonstrated the potential of auto-encoder compared with the three other related methods.

Second, it is worth noting that our cylindrical histogram provides a good visual understanding (see Fig. 3) while intermediate features extracted from a cloud-oriented deep neural network would be much more difficult to

Table 6 EERs obtained from experiments on two additional datasets

\begin{tabular}{lllllll}
\hline \multirow{2}{*}{ Method } & \multicolumn{2}{c}{ CMU } & & \multicolumn{2}{c}{ SFU } \\
\cline { 2 - 3 } \cline { 6 - 7 } & Frame & Sequence & & Frame & Sequence \\
\hline K-means [6] & - & 0.133 & & - & 0.474 \\
Bayesian GMM [6] & - & 0.133 & & - & 0.231 \\
One-class SVM [25] & - & 0.400 & & - & 0.356 \\
Bayesian GMM [25] & - & 0.267 & & - & 0.350 \\
Ours (leaky ReLU) & 0.233 & 0.067 & & 0.253 & 0.158 \\
\hline
\end{tabular}

The two methods $[6,25]$ are not adaptive to perform per-frame assessment The italic values indicate the best results interpret. Therefore, our method is more appropriate for practical applications where users/operators are not familiar with the more difficult interpretation of intermediate features in deep networks.

Another important factor is the coordinate system that is illustrated in Fig. 3. A setting that does not satisfy this constraint might significantly affect the ability of extracted histograms in reasonably representing gait postures. In that case, a rigid transformation [12] is an appropriate solution to guarantee the constraint.

Finally, the local motion of body parts (e.g., limbs) is not explicitly considered in a sequence of cylindrical histograms. A further investigation of such local descriptions is expected to increase the applicability of the method to specific gait problems.

\section{Conclusion}

This paper proposes an approach that estimates the gait normality index based on a sequence of point clouds formed by a ToF depth camera and two mirrors. Using such system not only reduces the price of devices but also avoids the requirement of a synchronization protocol since the data acquisition is performed by only one camera. This work introduces a simple hand-crafting feature, cylindrical histogram, extracted from raw input clouds that efficiently represents characteristics of walking postures. Auto-encoders with a specific block-level depth and various block structures are then employed to process such sequence of histograms, and the resulting losses are considered as gait normality indices. The efficiency of our method was demonstrated in the experiments using a dataset of 9 subjects with 9 different walking gaits. The quality of 3D point clouds provided by our setup was also highlighted in a comparison with other related works that employed different input data types (skeleton, silhouette, and depth map). Our method could be appropriate for many gait-related tasks such as assessing patient recovery after a lower limb surgery for instance.

In further works, elaborate experiments will be performed to select the block that is best appropriate with our model structure. Besides, sparsity constraints will be considered to give visual understanding about characteristics embedded inside the cylindrical histograms that are useful for gait-related tasks. Finally, modeling specific pathological gaits using our auto-encoders is also an interesting future study.

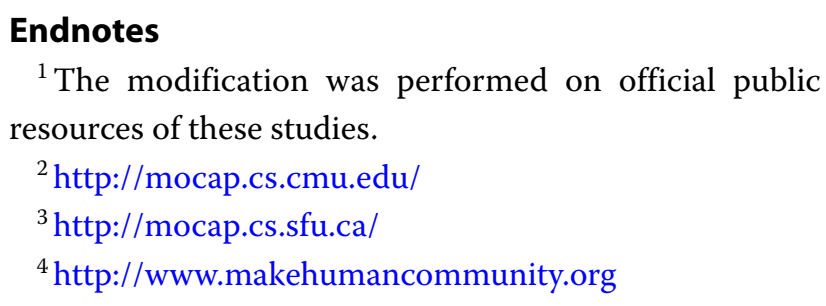

Endnotes

${ }^{1}$ The modification was performed on official public resources of these studies.

${ }^{2}$ http://mocap.cs.cmu.edu/

${ }^{3}$ http://mocap.cs.sfu.ca/

${ }^{4}$ http://www.makehumancommunity.org 


\section{Abbreviations}

AUC: Area under curve; EER: Equal error rate; HMM: Hidden Markov model; MSE: Mean square error; ReLU: Rectified linear unit; ROC: Receiver operating characteristic; SVM: Support vector machine; ToF: Time-of-flight

\section{Acknowledgements}

We would like to thank Hoang Anh Nguyen (Aeva Inc., Mountain View, CA, USA) for useful discussions. This project used data adapted from (1) mocap.cs.cmu.edu created with funding from NSF EIA-0196217 and (2) mocap.cs.sfu.ca created with funding from NUS ACRF R-252-000-429-133 and SFU President's Research Start-up Grant.

\section{Funding}

Financial support for this work was provided by the Natural Sciences and Engineering Research Council of Canada (NSERC) under Discovery Grant RGPIN-2015-05671.

\section{Availability of data and materials}

The dataset analyzed during the current study is available in the DIRO Vision lab repository, http://www-labs.iro.umontreal.ca/ labimage/GaitDataset Prepared (normalized) cylindrical histograms of size $16 \times 16$ are available at http://www-labs.iro.umontreal.ca/ labimage/GaitDataset/normhists.zip (unzip password: wUz7EcH9xG)

A Python implementation of this work will be available on Github upon the publication of the manuscript.

Two additional datasets used in our experiments are available at http://wwwlabs.iro.umontreal.ca/ labimage/AdditionalGaitSets.

\section{Authors' contributions}

TNN carried out the work, designed the experiments, and drafted the manuscript. JM has supervised the work. All authors read and approved the final manuscript.

\section{Competing interests}

The authors declare that they have no competing interests.

\section{Publisher's Note}

Springer Nature remains neutral with regard to jurisdictional claims in published maps and institutional affiliations.

\section{Received: 7 September 2018 Accepted: 8 May 2019}

Published online: 28 May 2019

\section{References}

1. M. Abadi, P. Barham, J. Chen, Z. Chen, A. Davis, J. Dean, M. Devin, S. Ghemawat, G. Irving, M. Isard, M. Kudlur, J. Levenberg, R. Monga, S. Moore, D. G. Murray, B. Steiner, P. Tucker, V. Vasudevan, P. Warden, M. Wicke, Y. Yu, $X$. Zheng, in Proceedings of the 12th USENIX Conference on Operating Systems Design and Implementation, USENIX Association, Berkeley, CA, USA, OSDI'16. Tensorflow: a system for large-scale machine learning, (2016), pp. 265-283. http://dl.acm.org/citation.cfm?id=3026877.3026899

2. N. Ali, K. B. Bajwa, R. Sablatnig, S. A. Chatzichristofis, Z. Iqbal, M. Rashid, H. A. Habib, A novel image retrieval based on visual words integration of sift and surf. PLoS ONE. 11(6), 1-20 (2016). https://doi.org/10.1371/ journal.pone.0157428

3. N. Ali, B. Zafar, F. Riaz, S. Hanif Dar, N. lqbal Ratyal, K. Bashir Bajwa, M. Kashif lqbal, M. Sajid, A hybrid geometric spatial image representation for scene classification. PLoS ONE. 13(9), 1-27 (2018). https://doi.org/10.1371/ journal.pone.0203339

4. C. Bauckhage, J. K. Tsotsos, F. E. Bunn, in The 2nd Canadian Conference on Computer and Robot Vision (CRV'05). Detecting abnormal gait, (2005), pp. 282-288. https://doi.org/10.1109/CRV.2005.32

5. C. Bauckhage, J. K. Tsotsos, F. E. Bunn, Automatic detection of abnormal gait. Image Vis. Comput. 27(1), 108-115 (2009)

6. S. Bei, Z. Zhen, Z. Xing, L. Taocheng, L. Qin, Movement disorder detection via adaptively fused gait analysis based on kinect sensors. IEEE Sensors J. 18(17), 7305-7314 (2018). https://doi.org/10.1109/JSEN.2018.2839732

7. Y. Bengio, P. Lamblin, D. Popovici, H. Larochelle, in Proceedings of the 19th International Conference on Neural Information Processing Systems, MIT Press, Cambridge, MA, USA, NIPS'06. Greedy layer-wise training of deep networks (MIT Press, Cambridge, MA, 2006), pp. 153-160
8. A. A. M. Bigy, K. Banitsas, A. Badii, J. Cosmas, in 2015 7th International IEEE/EMBS Conference on Neural Engineering (NER). Recognition of postures and freezing of gait in Parkinson's disease patients using Microsoft Kinect sensor, (2015), pp. 731-734. https://doi.org/10.1109/NER.2015.7146727

9. J. W. Davis, Hierarchical motion history images for recognizing human motion. (Proceedings IEEE Workshop on Detection and Recognition of Events in Video, 2001), pp. 39-46

10. X. Glorot, Y. Bengio, in Proceedings of the Thirteenth International Conference on Artificial Intelligence and Statistics, PMLR, Chia Laguna Resort, Sardinia, Italy, Proceedings of Machine Learning Research, vol. 9, ed. by Y.W. Teh, M. Titterington. Understanding the difficulty of training deep feedforward neural networks (PMLR, 2010), pp. 249-256

11. J. Han, B. Bhanu, Individual recognition using gait energy image. IEEE Trans. Pattern Anal. Mach. Intell. 28(2), 316-322 (2006). https://doi.org/10. 1109/TPAMI.2006.38

12. R. Hartley, A. Zisserman, Multiple view geometry in computer vision. (Cambridge university press, New York, NY, 2003)

13. G. E. Hinton, S. Osindero, Y. W. Teh, A fast learning algorithm for deep belief nets. Neural Comput. 18(7), 1527-1554 (2006). https://doi.org/10. 1162/neco.2006.18.7.1527

14. Q. Huang, W. Wang, U. Neumann, in The IEEE Conference on Computer Vision and Pattern Recognition (CVPR). Recurrent slice networks for 3D segmentation of point clouds (IEEE, 2018)

15. Y. Jia, E. Shelhamer, J. Donahue, S. Karayev, J. Long, R. Girshick, S. Guadarrama, T. Darrell, in Proceedings of the 22Nd ACM International Conference on Multimedia, ACM, New York, NY, USA, MM'14. Caffe: Convolutional architecture for fast feature embedding, (2014), pp. 675-678. http://doi.acm.org/10.1145/2647868.2654889

16. S. Jiang, Y. Wang, Y. Zhang, J. Sun, Real Time Gait Recognition System Based on Kinect Skeleton Feature. (Springer International Publishing, Cham, 2015), pp. 46-57

17. Z. Lv, X. Xing, K. Wang, D. Guan, Class energy image analysis for video sensor-based gait recognition: a review. Sensors. 15(1), 932-964 (2015). https://doi.org/10.3390/s150100932. http://www.mdpi.com/1424-8220/ $15 / 1 / 932$

18. A. L. Maas, A. Y. Hannun, A. Y. Ng, in Proc. ICML, vol 30. Rectifier nonlinearities improve neural network acoustic models (PMLR, 2013)

19. T. N. Nguyen, J. Meunier, Walking gait dataset: point clouds, skeletons and silhouettes. Tech. Rep 1379, DIRO, University of Montreal, (2018). http://www. iro.umontreal.ca/ labimage/GaitDataset/dataset.pdf

20. T. N. Nguyen, H. H. Huynh, J. Meunier, in Proceedings of the Fifth Symposium on Information and Communication Technology, ACM, New York, NY, USA, SolCT'14. Extracting silhouette-based characteristics for human gait analysis using one camera, (2014), pp. 171-177. http://doi. acm.org/10.1145/2676585.2676612

21. T. N. Nguyen, H. H. Huynh, J. Meunier, Skeleton-based abnormal gait detection. Sensors. 16(11), 1792 (2016). https://doi.org/10.3390/ s16111792. http://www.mdpi.com/1424-8220/16/11/1792

22. T. N. Nguyen, H. H. Huynh, J. Meunier, 3D reconstruction with time-of-flight depth camera and multiple mirrors. IEEE Access. 6 , 38,106-38,114 (2018a). https://doi.org/10.1109/ACCESS.2018.2854262

23. T. N. Nguyen, H. H. Huynh, J. Meunier, in 2018 IEEE EMBS International Conference on Biomedical Health Informatics (BHI), Las Vegas, NV, USA. Assessment of gait normality using a depth camera and mirrors, (2018b), pp. 37-41. https://doi.org/10.1109/BHI.2018.8333364

24. C. R. Qi, H. Su, K. Mo, L. J. Guibas, in The IEEE Conference on Computer Vision and Pattern Recognition (CVPR). Pointnet: deep learning on point sets for 3D classification and segmentation (IEEE, 2017)

25. P. Ren, S. Tang, F. Fang, L. Luo, L. Xu, M. L. Bringas-Vega, D. Yao, K. M. Kendrick, P. A. Valdes-Sosa, Gait rhythm fluctuation analysis for neurodegenerative diseases by empirical mode decomposition. IEEE Trans. Biomed. Eng. 64(1), 52-60 (2017). https://doi.org/10.1109/TBME.2016.2536438

26. T. N. Sainath, B. Kingsbury, B. Ramabhadran, in 2012 IEEE International Conference on Acoustics, Speech and Signal Processing (ICASSP). Auto-encoder bottleneck features using deep belief networks, (2012), pp. 4153-4156. https://doi.org/10.1109/ICASSP.2012.6288833

27. M. Sajid, N. Ali, S. H. Dar, N. lqbal Ratyal, A. R. Butt, B. Zafar, T. Shafique, M. J. A. Baig, I. Riaz, S. Baig, Data augmentation-assisted makeup-invariant face recognition. Math Probl. Eng. 2018 (2018)

28. S. Shi, Q. Wang, P. Xu, X. Chu, in 20167 th International Conference on Cloud Computing and Big Data (CCBD). Benchmarking state-of-the-art deep 
learning software tools, (2016), pp. 99-104. https://doi.org/10.1109/CCBD 2016.029

29. J. Shotton, A. Fitzgibbon, M. Cook, T. Sharp, M. Finocchio, R. Moore, A. Kipman, A. Blake, in CVPR, vol. 2011. Real-time human pose recognition in parts from single depth images, (2011), pp. 1297-1304. https://doi.org/10. 1109/CVPR.2011.5995316

30. J. Shotton, R. Girshick, A. Fitzgibbon, T. Sharp, M. Cook, M. Finocchio, R Moore, P. Kohli, A. Criminisi, A. Kipman, A. Blake, Efficient human pose estimation from single depth images. IEEE Trans. Pattern Anal. Mach. Intell. 35(12), 2821-2840 (2013). https://doi.org/10.1109/TPAMI.2012.24

31. S. Smeureanu, R. T. Ionescu, M. Popescu, B. Alexe, in Image Analysis and Processing - ICIAP, vol. 2017, ed. by S. Battiato, G. Gallo, R. Schettini, and F. Stanco. Deep appearance features for abnormal behavior detection in video (Springer International Publishing, Cham, 2017), pp. 779-789

32. N. Srivastava, G. Hinton, A. Krizhevsky, I. Sutskever, R. Salakhutdinov, Dropout: a simple way to prevent neural networks from overfitting. J. Mach. Learn. Res. 15, 1929-1958 (2014). http://jmlr.org/papers/v15/ srivastava14a.html

33. T. Tieleman, G. Hinton, Lecture 6.5-rmsprop: divide the gradient by a running average of its recent magnitude. COURSERA: Neural Netw. Mach. Learn. 4(2), 26-31 (2012)

34. J. S. Vitter, Faster methods for random sampling. Commun ACM. 27(7), 703-718 (1984). https://doi.org/10.1145/358105.893. http://doi.acm.org/ $10.1145 / 358105.893$

35. B. Wang, X. Liu, K. Xia, K. Ramamohanarao, D. Tao, Random angular projection for fast nearest subspace search. (R. Hong, W. H. Cheng, T. Yamasaki, M. Wang, C. W. Ngo, eds.) (Springer International Publishing, Cham, 2018), pp. 15-26

36. X. Wang, L. Gao, P. Wang, X. Sun, X. Liu, Two-stream 3-D convNet fusion for action recognition in videos with arbitrary size and length. IEEE Trans. Multimed. 20(3), 634-644 (2018). https://doi.org/10.1109/TMM.2017. 2749159

37. Wolfram Research Inc, Mathematica, Version 11.1. Champaign, IL, 2017 (2017). http://support.wolfram.com/kb/41360

38. K. Xia, Y. Ma, X. Liu, Y. Mu, L. Liu, in Proceedings of the 25th ACM International Conference on Multimedia, ACM, New York, NY, USA, MM'17. Temporal binary coding for large-scale video search, (2017), pp. 333-341. https://doi.org/10.1145/3123266.3123273

39. Y. Yang, C. Feng, Y. Shen, D. Tian, in The IEEE Conference on Computer Vision and Pattern Recognition (CVPR). Foldingnet: point cloud auto-encoder via deep grid deformation (IEEE, 2018)

40. B. Zafar, R. Ashraf, N. Ali, M. Ahmed, S. Jabbar, S. A. Chatzichristofis, Image classification by addition of spatial information based on histograms of orthogonal vectors. PLOS ONE. 13(6), 1-26 (2018a). https://doi.org/10. 1371/journal.pone.0198175

41. B. Zafar, R. Ashraf, N. Ali, M. Ahmed, S. Jabbar, K. Naseer, A. Ahmad, G. Jeon, Intelligent image classification-based on spatial weighted histograms of concentric circles. Comput. Sci. Inf. Syst. 15(3), 615-633 (2018b). http://doiserbia.nb.rs/Article.aspx?id=1820-02141800025Z

42. B. Zafar, R. Ashraf, N. Ali, M. K. Iqbal, M. Sajid, S. H. Dar, N. I. Ratyal, A novel discriminating and relative global spatial image representation with applications in CBIR. Appl. Sci. 8(11) (2018b). https://doi.org/10.3390/ app8112242. http://www.mdpi.com/2076-3417/8/11/2242

\section{Submit your manuscript to a SpringerOpen ${ }^{\circ}$ journal and benefit from:}

- Convenient online submission

- Rigorous peer review

- Open access: articles freely available online

- High visibility within the field

- Retaining the copyright to your article

Submit your next manuscript at $\$$ springeropen.com 